\title{
Quantitative Variation in Effector Activity of ToxA Isoforms from Stagonospora nodorum and Pyrenophora tritici-repentis
}

\author{
Kar-Chun Tan, ${ }^{1}$ Margo Ferguson-Hunt, ${ }^{2}$ Kasia Rybak, ${ }^{2}$ Ormonde D. C. Waters, ${ }^{1}$ Will A. Stanley, ${ }^{3}$ \\ Charles S. Bond, ${ }^{4}$ Eva H. Stukenbrock, ${ }^{5}$ Timothy L. Friesen, ${ }^{6}$ Justin D. Faris, ${ }^{6}$ Bruce A. McDonald, ${ }^{7}$ \\ and Richard P. Oliver ${ }^{1}$ \\ ${ }^{1}$ Environment and Agriculture, Curtin University, Bentley WA 6102, Australia; ${ }^{2} \mathrm{Health}$ Science, Murdoch University, Murdoch, \\ WA 6150, Australia; ${ }^{3}$ ARC Centre of Excellence in Plant Energy Biology and ${ }^{4}$ Biomedical, Biomolecular and Chemical \\ Sciences, The University of Western Australia, Crawley 6009 WA, Australia; ${ }^{5}$ Max Planck Institute Marburg, Karl von Frisch \\ Str. 10, D-35043 Marburg, Germany; ${ }^{6}$ United States Department of Agriculture-Agricultural Research Service Cereal Crops \\ Research Unit, Red River Valley Agricultural Research Center, Fargo, ND 58105, U.S.A.; ${ }^{7}$ Plant Pathology Group, Institute \\ of Integrative Biology, ETH Zurich, Universitätsstr 2, CH-8092 Zurich, Switzerland
}

Submitted 25 October 2011. Accepted 12 December 2011.

ToxA is a proteinaceous necrotrophic effector produced by Stagonospora nodorum and Pyrenophora tritici-repentis. In this study, all eight mature isoforms of the ToxA protein were purified and compared. Circular dichroism spectra indicated that all isoforms were structurally intact and had indistinguishable secondary structural features. ToxA isoforms were infiltrated into wheat lines that carry the sensitivity gene $T s n 1$. It was observed that different wheat lines carrying identical Tsn1 alleles varied in sensitivity to ToxA. All ToxA isoforms induced necrosis when introduced into any $T s n 1$ wheat line but we observed quantitative variation in effector activity, with the least-active version found in isolates of $P$. tritici-repentis. Pathogen sporulation increased with higher doses of ToxA. The isoforms that induced the most rapid necrosis also induced the most sporulation, indicating that pathogen fitness is affected by differences in ToxA activity. We show that differences in toxin activity encoded by a single gene can contribute to the quantitative inheritance of necrotrophic virulence. Our findings support the hypothesis that the variation at $\operatorname{Tox} A$ results from selection that favors increased toxin activity.

Microbial plant pathogens interact with their hosts via the interplay of numerous pathogen molecules and plant-interacting partners. Many of these molecules are relatively invariant components of pathogens that are recognized by the host and induce a defense response; these are referred to as pathogenor microbe-associated molecular patterns (Chisholm et al. 2006; De Wit et al. 2009; Jones and Dangl 2006). Other molecules (now referred to as effectors) suppress defense responses induced by plant recognition of pathogen-associated molecular patterns (Jones and Dangl 2006). Suppression of host defense responses, otherwise known as effector-triggered susceptibility (ETS) (Jones and Dangl 2006), can be countered by evolution of the host of a receptor that recognizes the effector, leading to a defense response called effector-triggered immunity (ETI).

Corresponding author: R. Oliver; E-mail: richard.oliver@curtin.edu.au

* The $\boldsymbol{e}$-Xtra logo stands for "electronic extra" and indicates four supplementary figures and one supplementary table are published online.
One consequence of ETS and ETI is an escalating co-evolutionary arms race mediated by accelerated selection of diversified effector and interactor gene sequences. The hallmark of this co-evolution is diversifying selection, leading to multiple alleles of the effectors and interactors, as has been observed in pathogen systems as diverse as the fungal ascomycete Cladosporium fulvum (Stergiopoulos et al. 2007), the basidiomycete Melampsora lini (Dodds et al. 2006; Ellis et al. 2008), the oomycete Hyaloperonospora arabidopsis (Allen et al. 2004), as well as numerous bacterial systems (Abramovitch et al. 2006).

These concepts and findings have been derived almost exclusively by consideration of biotrophic host interactions (Dodds et al. 2009; Lewis 1973; Oliver and Ipcho 2004). Biotrophy is characterized by the absence of significant host necrosis in compatible interactions and essentially complete resistance in incompatible interactions (Poland et al. 2009). Necrotrophic pathogens also produce specialized effectors (necrotrophic effectors [NE], formerly called host-specific or host-selective toxins) that induce necrotic reactions in a host- and cultivarspecific manner. The parallels between the effector-host interactions in biotrophic and necrotrophic interactions are striking (Wolpert et al. 2002) and it has recently been confirmed that NE, like biotrophic avirulence effectors, also interact (directly or indirectly) with receptor-like proteins encoded by nucleotide-binding site leucine-rich repeat (LRR) genes (Lorang et al. 2007; Nagy and Bennetzen 2008; Sweat et al. 2008). Nonetheless, there is a clear functional distinction. Whereas biotrophic pathogens can be said to benefit from the absence of recognition of avirulence or effector products, necrotrophic pathogens are at least partially dependent for virulence on positive recognition of their NE (Friesen et al. 2008; Leach et al. 1982; Oliver and Solomon 2010; Yoder 1980). Furthermore, necrotrophic disease interactions are typically quantitative, with resistance being partial and characterized by many quantitative trait loci (Poland et al. 2009).

Two important pathogens of wheat (Triticum aestivum L.) worldwide are the necrotrophs Stagonospora (syn. Septoria or Phaeosphaeria) nodorum and Pyrenophora tritici-repentis that cause the diseases Stagonospora (syn. Septoria) nodorum (or glume) blotch and tan (syn. yellow [leaf]) spot, respectively (Lamari et al. 1998; Solomon et al. 2006a). Symptom develop- 
ment and appearance is very similar for both diseases. Pathogenicity in $P$. tritici-repentis has been associated with the production of three NE, of which ToxA is the best characterized (Friesen et al. 2002, 2003, 2008; Manning and Ciuffetti 2005; Manning et al. 2004, 2007; Rasmussen et al. 2004; Sarma et al. 2005; Tuori et al. 2000). ToxA is a 13.2-kDa secreted protein encoded by a single gene, which causes necrosis in wheat cultivars carrying an active allele of $T s n l$. Fungal strains that express the ToxA gene are significantly more virulent on $T s n 1$ wheat lines. Conversely, wheat lines that lack Tsn1 (i.e., carry the recessive allele $t s n 1$ ) are significantly more resistant to ToxA-expressing fungal strains. Therefore, ToxA can be regarded as an important effector of $P$. tritici-repentis virulence. ToxA has been reported to be internalized into mesophyll cells of Tsnl but not tsnl wheat lines, after which it interacts with a ubiquitous chloroplast protein, ToxABP (Manning and Ciuffetti 2005; Manning et al. 2007). It has also been reported to interact with plastocyanin (Tai et al. 2007). Both interactions are consistent with the observation that necrosis is light dependent.

The role of NE in the virulence of $S$. nodorum has emerged more recently (Friesen et al. 2007, 2008; Liu et al. 2004a,b; Oliver 2008; Oliver et al. 2008, 2009). S. nodorum appears to express at least five and possibly several more NE genes (Abeysekara et al. 2009; Friesen et al. 2007, 2008, 2009; Liu et al. 2009; Zhang et al. 2011). Elucidation of the genome sequence of an Australian isolate of $S$. nodorum revealed that it produced a version of ToxA that differed at just four nucleotides from $P$. tritici-repentis ToxA (Friesen et al. 2006). As in $P$. tritici-repentis, expression of ToxA contributes to $S$. nodorum virulence, while sensitivity and susceptibility to the NE and the disease, respectively, is controlled by $T s n 1$ (Faris and Friesen 2009; Liu et al. 2006).

Examination of large collections of fungal strains revealed important differences between the species with respect to ToxA. Approximately $80 \%$ of $P$. tritici-repentis isolates but only $40 \%$ of $S$. nodorum isolates carried ToxA genes (Friesen et al. 2006; Stukenbrock and McDonald 2007). No polymorphisms were found in ToxA in any of the 54 global $P$. triticirepentis isolates analyzed (Friesen et al. 2006). In contrast, 21 polymorphic sites were revealed when $123 \mathrm{~S}$. nodorum ToxA genes were sequenced (Stukenbrock and McDonald 2007). These 21 polymorphisms resolved as 13 haplotypes and 11 full-length predicted amino acid sequences (isoforms). One isoform (H3) included two premature stop codons, suggesting that the allele is nonfunctional (Stukenbrock and McDonald 2007). Removal of the pre- and pro-sequences left seven different mature protein isoforms. Examination of the $S$. nodorum and $P$. tritici-repentis ToxA gene sequences identified two sites with elevated nonsynonymous over synonymous $(\mathrm{dN} / \mathrm{dS})$ ratios (Stukenbrock and McDonald 2007). High ratios have been observed in biotrophic effector and resistance genes undergoing diversifying selection. Thus, the $\mathrm{dN} / \mathrm{dS}$ ratio was consistent with a model where ToxA molecules interact directly with a functional plant interaction partner ("receptor"), suggesting that a co-evolutionary arms race might be operating. An alternative explanation for the observed diversity is that ToxA isoforms differ in activity and that selection has favored isoforms that increase pathogen fitness.

The wheat gene Tsnl governs sensitivity to ToxA and has recently been isolated by positional cloning (Faris et al. 2010). Tsnl encodes a 1,490-amino-acid protein that contains serine/ threonine protein kinase and LRR domains. Nearly all wheat cultivars that are insensitive to ToxA completely lack the gene; in 'Puseas' and 'Novo' wheat, the gene is present but nonsense mutations render the protein nonfunctional. All active breadwheat copies of $T_{s n} 1$ encode an identical predicted amino acid sequence. The Tsn1 protein has not been shown to interact directly with ToxA (Faris et al. 2010).

The identification of eight isoforms of ToxA provides a unique opportunity to elucidate the quantitative nature of necrotrophic virulence. We carefully examined the interplay between the $S$. nodorum and $P$. tritici-repentis isoforms of ToxA and host lines carrying various sources of Tsn 1 . We investigated the functional relevance of the observed allelic variation for ToxA and compared it with the allelic variation found for effectors in biotrophic fungi. We show that all ToxA isoforms are recognized by all wheat cultivars that express $T s n 1$ but that there are significant quantitative differences in activity among the effector isoforms. The more active isoforms of ToxA induced more sporulation in a reconstituted infection assay. We asked whether these differences in activity can explain the quantitative nature of host-parasite interactions observed for $S$. nodorum (and other necrotrophic fungi). We hypothesized that the observed allelic variation resulted from selection that favors increased necrotrophic effector activity. These findings constitute a novel molecular explanation for the quantitative nature of disease interactions involving necrotrophic fungi.

\section{RESULTS}

\section{Biochemical characterization of ToxA isoforms.}

Production of each of the different effector isoforms (Table 1) was achieved through purification of his-tagged proteins heterologously expressed in Escherichia coli. All purified ToxA isoforms were checked for size and purity by gel electrophoresis (Fig. 1). All isoforms gave bands of the expected size and were highly purified. The proteins were subjected to circular dichroism (CD) spectroscopy to evaluate secondary structure and compared with the three-dimensional configuration of PtrToxA:H15 (Sarma et al. 2005). Secondary structure was found to be consistent among all isoforms and to coincide closely with the crystallized PtrToxA:H15 (Table 2) and, thus,

Table 1. Summary of amino-acid changes in the mature proteins of the ToxA isoforms ${ }^{\mathrm{a}}$

\begin{tabular}{|c|c|c|c|c|c|c|c|c|}
\hline \multirow[b]{2}{*}{ Nucleotide } & \multicolumn{8}{|c|}{ Position } \\
\hline & 297 & 310 & 340 & $361-2-3$ & 405 & 412 & 487 & 499 \\
\hline Amino acid & 99 & 104 & 114 & 121 & 135 & 138 & 163 & 167 \\
\hline Structure & $\beta 2$ & $\beta 3$ & $\beta 3-\beta 4$ & $\beta 4$ & $\beta 5-\beta 6$ & $\beta 5-\beta 6$ & $\beta 7$ & $\beta 7-\beta 8$ \\
\hline$\overline{\mathrm{H} 2}$ & $\mathrm{E}$ & $\mathrm{V}$ & $\mathrm{N}$ & $\mathrm{N}$ & $\mathrm{E}$ & $\mathrm{L}$ & I & $\mathrm{S}$ \\
\hline $\mathrm{H} 4$ & $\mathrm{D}$ & I & $\mathrm{N}$ & $\mathrm{N}$ & $\mathrm{E}$ & $\mathrm{L}$ & I & $\mathrm{S}$ \\
\hline H6 & $\mathrm{E}$ & I & $\mathrm{N}$ & V & $\mathrm{E}$ & $\mathrm{L}$ & I & $\mathrm{S}$ \\
\hline $\mathrm{H} 8$ & $\mathrm{D}$ & I & $\mathrm{N}$ & $\mathrm{N}$ & $\mathrm{E}$ & $\mathrm{L}$ & $\mathrm{L}$ & $\mathrm{S}$ \\
\hline H9 & $\mathrm{E}$ & I & $\mathrm{N}$ & $\mathrm{N}$ & $\mathrm{E}$ & $\mathrm{L}$ & I & $\mathrm{S}$ \\
\hline H12 & $\mathrm{E}$ & V & $\mathrm{N}$ & V & $\mathrm{E}$ & $\mathrm{L}$ & I & $\mathrm{S}$ \\
\hline H13 & $\mathrm{E}$ & I & $\mathrm{N}$ & $\mathrm{N}$ & $\mathrm{E}$ & $\mathrm{L}$ & $\mathrm{L}$ & $\mathrm{S}$ \\
\hline H15 (PtrToxA) & $\mathrm{E}$ & I & $\mathrm{D}$ & $\mathrm{R}$ & $\mathrm{D}$ & V & I & $\mathrm{T}$ \\
\hline
\end{tabular}

a Pro-protein starts at amino-acid position 60; nucleotide 180. H3 includes two stop codons and is inactive. 
the toxin isoforms were judged to be correctly folded and not globally altered in structure by variation in sequence.

\section{Effector activity of ToxA isoforms.}

Each of the isoforms of S. nodorum ToxA (SnToxA:H2, H4, $\mathrm{H} 6, \mathrm{H} 8, \mathrm{H} 9, \mathrm{H} 12$, and $\mathrm{H} 13$ ) and $P$. tritici-repentis ToxA (PtrToxA:H15) was infiltrated into wheat leaves and incubated as described. Initially, the proteins were infiltrated into 'BR34' (tsn1), 'Grandin', and a line that carries only $T s n 1$, the SnToxA differential line 'BG261' (Friesen et al. 2006) (Fig. 2). Recessive $t s n 1$ lines were previously shown to be insensitive to ToxA from $P$. tritici-repentis and from the sequenced isolate of $S$. nodorum, SnToxA:H1 (an isoform identical to H9) (Friesen et al. 2006, 2007; Liu et al. 2006). Each isoform induced obvious necrosis in the Tsn1 lines and not in BR34. We further checked a series of $T s n 1$ wheat lines ('Glenlea', 'Katepwa', Grandin, 'ND2709', 'Sumai3', 'ND495', and 'Timstein') (Faris et al. 1996) that included a wide range of genetic backgrounds but carried identical Tsn 1 protein coding sequences. Also used were two PtrToxA-insensitive lines, Puseas and Novo. When infiltrated at $100 \mu \mathrm{g} / \mathrm{ml}$, all ToxA isoforms gave necrotic reactions on the previously designated Tsnl wheat lines (Fig. 2). No reaction was observed with the tsnl lines even with the highest concentrations of extracts. This indicates that the mutations distinguishing the eight fulllength ToxA isoforms did not prevent recognition by the products of the Tsnl alleles present in these wheat lines. An inactive version of ToxA (H3) (Stukenbrock and McDonald 2007) which includes several stop codons and an empty vector con- trol were also expressed in E. coli. No ToxA band was produced, as expected (data not shown). The E. coli extracts were infiltrated into all the wheat lines and produced no response. This confirmed the specificity of ToxA reaction on the selected wheat varieties.

In order to make a quantitative measure of the interactions between the ToxA isoforms and the different sources of Tsn1, the ToxA isoforms were carefully quantified and $25 \mu \mathrm{g} / \mathrm{ml}$ was infiltrated into the seven wheat lines. The infiltrations were carried out at the same time and the whole experiment was repeated at least three times. A representative assay is shown in supplemental data (Supplementary Fig. S2). The necrotic reaction was scored at 3 days according to a visual score of 0 to 4 (discussed below). The average necrosis score for each ToxA isoform and each cultivar is shown in Table 3.

We observed significant quantitative variation in both effector activity and receptor sensitivity. The scores for each effector were averaged over all seven wheat cultivars and statistically analyzed. $\mathrm{H} 4$ and $\mathrm{H} 2$ were consistently the most active effectors; H12, H6, H8, and H9 formed a middle group; and H13 and, especially, H15 were the weakest. Interestingly, the least active protein was PtrToxA:H15, with an average score only half that of $\mathrm{H} 2$ and $\mathrm{H} 4$ (Table 3). The scores for each ToxA version against individual cultivars are shown in supplementary data (Supplementary Fig. S3). There were no reproducible differences in effector rank order with any cultivars. In all cases, $\mathrm{H} 2$ and $\mathrm{H} 4$ were the most active and $\mathrm{H} 15$ was the least. The necrosis scores for each wheat line when infiltrated with all ToxA isoforms were averaged and statistically analyzed (Table 3 ). The

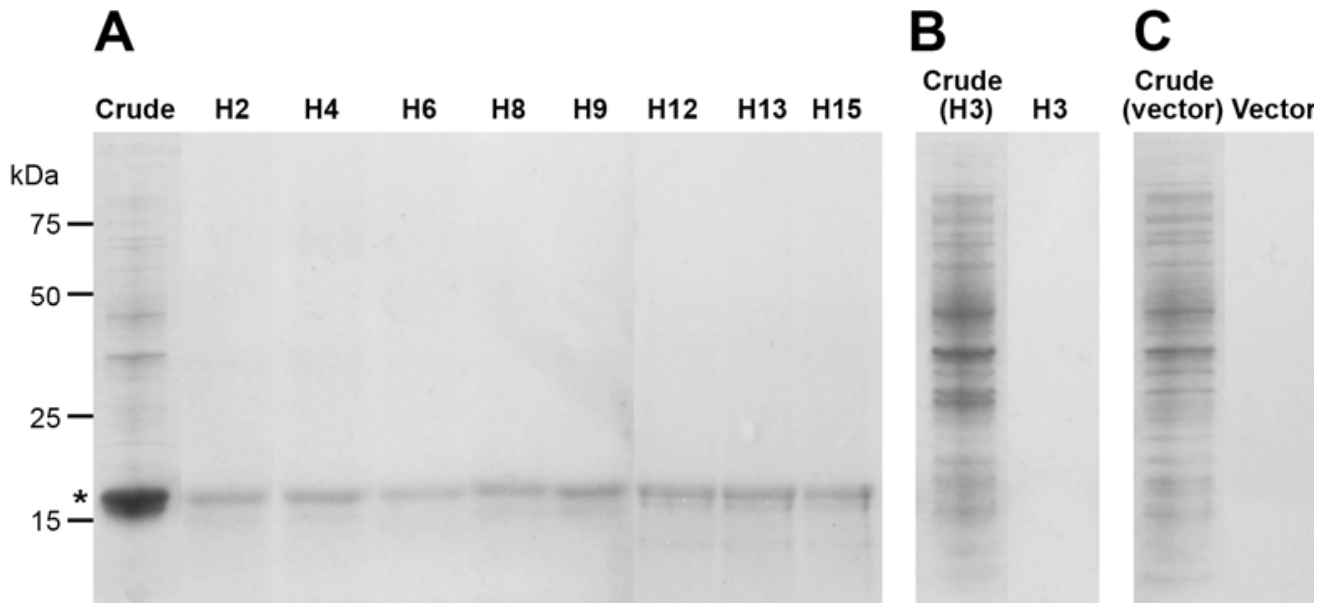

Fig. 1. A, Visualization of a representative Escherichia coli crude lysate containing ToxA (20 $\mu \mathrm{g})$ and purified his-tagged ToxA isoforms $(4 \mu \mathrm{g})$ via sodium dodecyl sulfate polyacrylamide gel electrophoresis. B, E. coli crude lysate expressing the attenuated ToxA H3 isoform (20 $\mu \mathrm{g})$ and attempted his-tag purification of H3. C, Crude lysate from an E. coli empty vector control $(20 \mu \mathrm{g})$ and attempted his-tag purification. Asterisk (*) denotes the main 17.9-kDa ToxA pro-protein band. The figure was compiled from multiple images taken of gels that were electrophoresed concurrently.

Table 2. Circular dichroism spectral characterization of ToxA isoforms ${ }^{\mathrm{a}}$

\begin{tabular}{lcccc}
\hline ToxA variant & $\boldsymbol{\alpha}^{\mathbf{b}}$ & $\boldsymbol{\beta}^{\mathbf{b}}$ & Other $^{\mathbf{b}}$ & NRMSD $^{\mathbf{c}}$ \\
\hline PtrToxA & \multicolumn{1}{c}{ N $^{\text {d }}$} & 54 & 41 & $\ldots$ \\
H2 & 5 & $38.2(1.07)$ & $53.5(0.76)$ & $0.38(0.05)$ \\
H4 & $8.3(0.70)$ & $38.8(0.32)$ & $52.4(0.40)$ & $0.16(0.01)$ \\
H6 & $8.9(0.10)$ & $38.0(0.12)$ & $52.5(0.45)$ & $0.13(0.01)$ \\
H8 & $9.4(0.55)$ & $37.9(0.85)$ & $52.4(0.21)$ & $0.14(0.02)$ \\
H9 & $9.6(1.05)$ & $38.7(0.58)$ & $51.0(0.32)$ & $0.20(0.03)$ \\
H12 & $10.2(0.55)$ & $40.9(0.66)$ & $52.9(0.69)$ & $0.44(0.04)$ \\
H13 & $6.2(0.89)$ & $38.6(0.25)$ & $52.5(0.38)$ & $0.14(0.01)$ \\
H15 (PtrToxA) & $9.0(0.50)$ & $38.8(0.72)$ & $53.5(0.12)$ & $0.23(0.09)$ \\
\hline
\end{tabular}

${ }^{a}$ All ToxA isoforms have secondary structure signatures consistent with the experimentally determined three-dimensional structure of PtrToxA:H15:ToxA.

${ }^{\mathrm{b}}$ Mean (standard deviation) secondary structure content over three samples, as estimated by CONTIN-LL.

${ }^{c}$ NRMSD $=$ normalized root-mean-square deviation.

${ }^{d}$ STRIDE assignment of secondary structure from the crystal structure of PtrToxA. 
range of scores was approximately twofold. Glenlea was the most sensitive cultivar and ND2709 was the least sensitive.

In order to evaluate the biological relevance of the variation in effector activity, we established a reconstituted virulence assay. Detached leaves of wheat cultivars carrying (BG261) or not carrying (BR34) Tsn1 were infected with the SN15::toxA strain, in which ToxA had been ablated (Friesen et al. 2006). Prior to inoculation, the leaves were infiltrated with selected ToxA isoforms with high, medium, and low activity (H4, H9, and H15, respectively). The leaves were examined 5 days later for sporulation. To quantify sporulation as it proceeds through its stages (Supplementary Fig. S4), pycnidia were classified and counted and a composite score calculated (as described below).

Sporulation by the SN15::toxA strain was most abundant and rapid when $\mathrm{H} 4$ was infiltrated at the highest concentration $(200 \mu \mathrm{g} / \mathrm{ml})$ into the ToxA-sensitive line BG261; a score of 151 was observed (Fig. 3; Table 4). A baseline sporulation score of approximately 20 was seen when $10 \mu \mathrm{g} / \mathrm{ml}$ or less was infiltrated. This is likely due to the expression of other effectors recognized by this wheat line. $\mathrm{H} 9$ and $\mathrm{H} 15$ at $200 \mu \mathrm{g} / \mathrm{ml}$ gave scores of 64 and 43, respectively, consistent with a quantitative relationship between sporulation and effector activity. Much lower sporulation was observed when the ToxA-insensitive line BR34 was infiltrated (score of 32 for $\mathrm{H} 4$ at $200 \mu \mathrm{g} / \mathrm{ml}$ ). The highest concentration of $\mathrm{H} 15$ gave a $T s n 1$-dependent sporulation similar to that induced by $\mathrm{H} 4$ at $25 \mu \mathrm{g} / \mathrm{ml}$, suggesting an approximately 10 -fold difference in specific activity between the most- and least-active effector isoforms.

\section{DISCUSSION}

Resistance to $S$. nodorum is a quantitative trait (Aguilar et al. 2005; Friesen et al. 2007; Liu et al. 2004a). Our results indicate that differences in activity of effector alleles encoded by the same gene can generate quantitative differences in virulence. This shows that the quantitative nature of the interaction can be explained (at least partially) by variation at a single pathogen gene. These experiments are the first to show significant differences in virulence among naturally occurring isoforms of a necrotrophic pathogen effector. We observed that the ToxA isoforms with the highest activity were associated
BR34

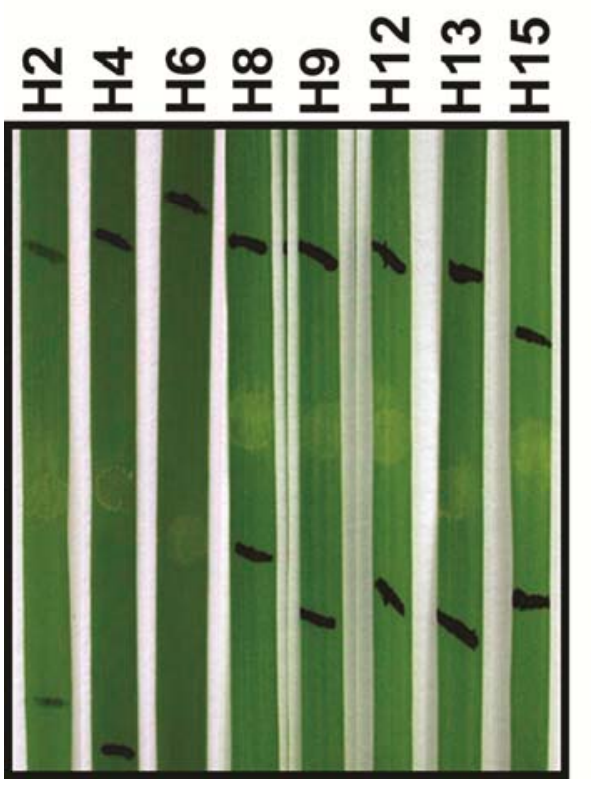

Grandin

\section{BG261}

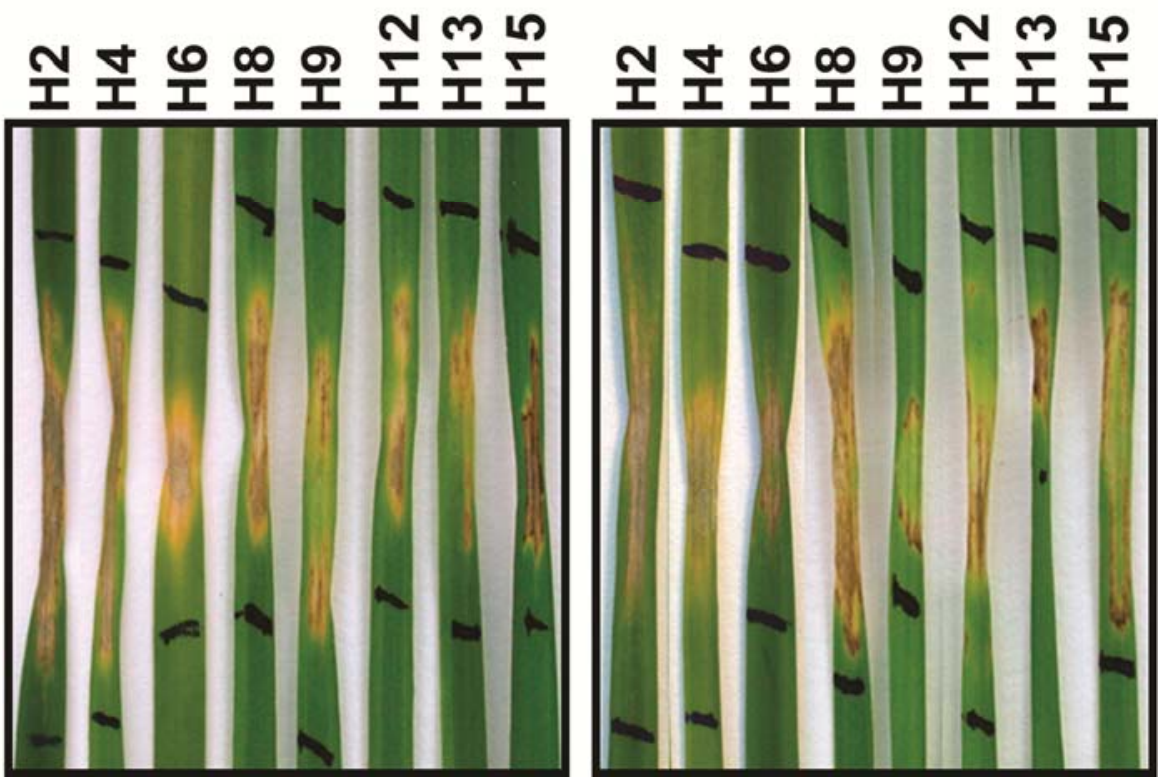

Fig. 2. Effect of selected ToxA isoforms on 'BR34' (tsn1), 'Grandin' (Tsn1). and 'BG261' (Tsn1). All ToxA forms induced necrosis on Tsnl but not on $t s n 1$ wheat cultivars.

Table 3. Average necrosis score of ToxA isoforms $(n=3)$ on seven $T s n 1$ wheat cultivars ${ }^{\mathrm{a}}$

\begin{tabular}{|c|c|c|c|c|c|c|c|c|c|c|}
\hline \multirow[b]{2}{*}{ Haplotype } & \multicolumn{7}{|c|}{ Wheat cultivar } & \multirow[b]{2}{*}{ Avg } & \multirow[b]{2}{*}{ SE } & \multirow[b]{2}{*}{ ANOVA grouping } \\
\hline & Glenlea & ND495 & Sumai3 & Katepwa & Timstein & Grandin & ND2709 & & & \\
\hline $\mathrm{H} 4$ & 4.0 & 4.0 & 4.0 & 4.0 & 4.0 & 4.0 & 4.0 & 4.0 & 0.00 & $\mathrm{a}$ \\
\hline $\mathrm{H} 2$ & 4.0 & 4.0 & 4.0 & 4.0 & 4.0 & 4.0 & 3.7 & 4.0 & 0.04 & $\mathrm{a}$ \\
\hline H12 & 4.0 & 4.0 & 4.0 & 4.0 & 3.7 & 3.7 & 2.7 & 3.7 & 0.18 & $a / b$ \\
\hline H6 & 4.0 & 4.0 & 4.0 & 3.7 & 4.0 & 4.0 & 2.0 & 3.7 & 0.28 & $a / b$ \\
\hline H8 & 4.0 & 4.0 & 1.0 & 3.7 & 4.0 & 4.0 & 1.0 & 3.1 & 0.54 & $a / b$ \\
\hline H9 & 4.0 & 3.0 & 4.0 & 3.3 & 1.7 & 2.0 & 1.0 & 2.7 & 0.44 & $a / b / c$ \\
\hline H13 & 4.0 & 3.0 & 3.3 & 2.0 & 2.0 & 1.3 & 1.0 & 2.4 & 0.41 & $\mathrm{~b} / \mathrm{c}$ \\
\hline H15 & 3.0 & 2.7 & 1.0 & 1.0 & 1.3 & 0.7 & 1.0 & 1.5 & 0.35 & c \\
\hline Avg & 3.9 & 3.5 & 3.0 & 3.1 & 3.0 & 2.8 & 1.8 & $\ldots$ & $\ldots$ & $\ldots$ \\
\hline $\mathrm{SE}$ & 0.13 & 0.21 & 0.50 & 0.41 & 0.43 & 0.51 & 0.38 & $\ldots$ & $\ldots$ & $\ldots$ \\
\hline ANOVA grouping & $\mathrm{a}$ & $\mathrm{a} / \mathrm{b}$ & $\mathrm{a} / \mathrm{b}$ & $\mathrm{a} / \mathrm{b}$ & $\mathrm{a} / \mathrm{b}$ & $\mathrm{a} / \mathrm{b}$ & $\mathrm{b}$ & $\ldots$ & $\ldots$ & $\ldots$ \\
\hline
\end{tabular}

${ }^{a}$ Infiltration with water, Escherichia coli expressing the inactive ToxA isoform $\mathrm{H} 3$ extract, and $E$. coli extract without ToxA (vector control) resulted in no observable symptoms (data not shown). Lowercase letters denote statistical groupings $(P<0.05)$ based on analysis of variance (ANOVA) Tukey-Kramer test to compare the average necrosis score for each ToxA isoform (vertical group) and wheat cultivars (horizontal group). Avg, average; SE, standard error of the mean. 
with the highest amount of pathogen reproduction, indicating that different isoforms could significantly affect pathogen fitness. Thus, selection favoring more virulent isoforms could explain the elevated ratio of $\mathrm{dN} / \mathrm{dS}$ found for ToxA.

Eight ToxA isoforms were cloned, expressed, and compared by CD spectroscopy. All displayed spectra consistent with the published X-ray crystal structure (Sarma et al. 2005). The variant amino acids were predominately conservative substitutions (E99D, I104V, D114N, E135D, L138V, I163L, and S167T); only one site, 121 , had widely differing amino acids $(\mathrm{N}, \mathrm{V}$, or $\mathrm{R})$. Furthermore, most changes were in surface-exposed loops between or at the termini of $\beta$-sheet regions (Supplementary Fig. S1). Notably, none of the variations affected sites previously shown to be critical for ToxA activity in experimentally derived mutants (Manning et al. 2004; Meinhardt et al. 2002; Sarma et al. 2005; Tuori et al. 2000).

One important result was that all isoforms of ToxA were active against all $T s n 1$-expressing wheat lines. Thus, all wheat lines shown previously to be sensitive to PtrToxA:H15 from $P$. tritici-repentis and the $\mathrm{H} 1$ form of ToxA from $S$. nodorum are likely to be sensitive to all isoforms of ToxA. This is in clear contrast to the situation with many avirulence gene products, where isoforms lack recognition by different plant receptors in a gene-for-gene manner (Ellis et al. 2006, 2008; Parlange et al. 2009; Stergiopoulos et al. 2007).

An equally important contrast to the biotrophic effector gene examples was the quantitative nature of the interaction between the ToxA isoforms and Tsnl wheat lines. Careful quantification of the ToxA preparations and multiple replications (the experiment has been carried out with four different sets of protein preparations and using a limiting dilution assay as well as the quantitative score recorded here) indicated a substantial range in both effector activity and varietal sensitivity. The biological relevance of the variation in effector activity was demonstrated in a reconstituted virulence activity. Sporulation was found to be ToxA-dose dependent, dependent on Tsnl expression, and significantly higher in $\mathrm{H} 4$ than $\mathrm{H} 9$ or $\mathrm{H} 15$, paralleling the necrotic activity (Fig. 3; Table 4).

The variation in activity among ToxA isoforms resulted in a twofold range in necrosis scores that reflects an approximate 10 -fold range in specific activity. The most active isoforms were $\mathrm{H} 4$ and $\mathrm{H} 2$. The $\mathrm{H} 4$ mature sequence is identical to $\mathrm{H} 5$ and H7. The next most active group was H6, H8, H9, and H12. The H9 mature sequence is identical to H1. H13 was significantly less active than $\mathrm{H} 2$ or $\mathrm{H} 4$. These $S$. nodorum isoforms shuffle mutations at four sites: E99D, V104I, N121V, and I163L. Two of these positions, 121 and 163, were earlier shown to exhibit an elevated $\mathrm{dN} / \mathrm{dS}$ ratio (Stukenbrock and McDonald 2007). The least-active isoform was the $P$. tritici-repentis ToxA isoform H15. Because H15 uniquely possesses D114, R121, D135, V138, and T167, it is not clear whether a single amino acid substitution or a combination is responsible for the lower activity. The biochemical basis of the difference in activity is not yet clear. There may be variation in resistance to proteolytic cleavage, in transport into the cytoplasm or chloroplast, or in binding to the plastid protein (Manning et al. 2007; Tai et al. 2007).

There was also a small but striking variation in sensitivity to all ToxA isoforms between different cultivars, with Glenlea being among the most sensitive and ND2709 the least. The amino acid sequence of the Tsn1 proteins in these cultivars is

Table 4. Reconstituted virulence assay showing composite virulence scores of Stagonospora nodorum toxA infection on wheat varieties infiltrated with selected ToxA isoforms

\begin{tabular}{llcc}
\hline & \multicolumn{3}{c}{ Isoform concentration $(\mu \mathrm{g} / \mathbf{m l})^{\mathbf{a}}$} \\
\cline { 2 - 4 } Variety, isoform & Buffer $(\mathbf{S E})$ & $\mathbf{2 5}(\mathbf{S E})$ & $\mathbf{2 0 0}(\mathrm{SE})$ \\
\hline BG261 & & & \\
H4 & $16.0(10.0)$ & $51.6(10.5)$ & $151.4(37.2)$ \\
H9 & $13.5(1.5)$ & $26.6(20.8)$ & $64.0(25.7)$ \\
H15 & $25.0(0.0)$ & $3.6(1.7)$ & $42.8(13.7)$ \\
BR34 & & & \\
H4 & $29.5(8.5)$ & $6.2(2.3)$ & $31.8(10.8)$ \\
H9 & $28.5(8.5)$ & $13.0(5.4)$ & $29.4(22.0)$ \\
H15 & $26.0(2.0)$ & $33.2(13.0)$ & $10.0(4.8)$ \\
\hline
\end{tabular}

${ }^{a} \mathrm{SE}$, standard error of the mean.

H4
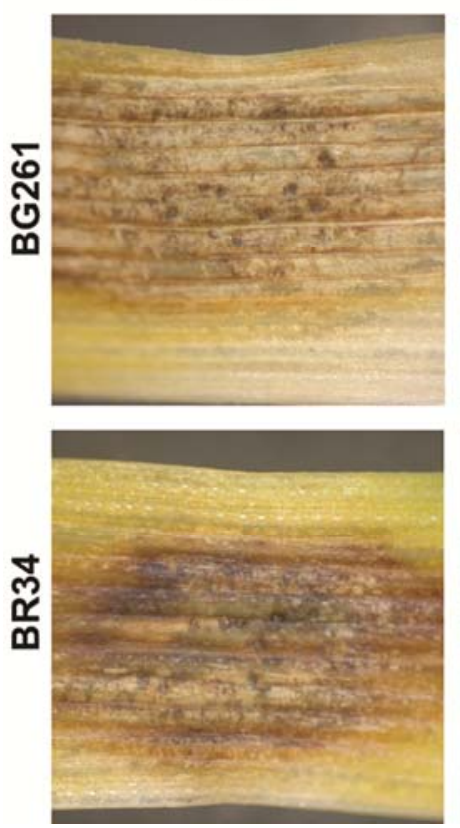
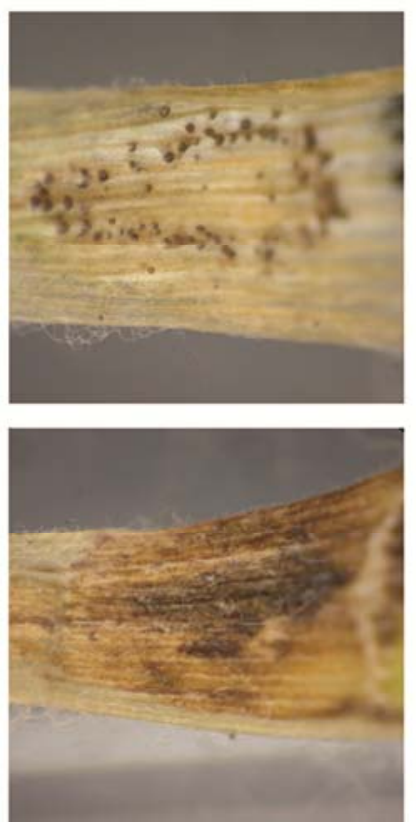

H9
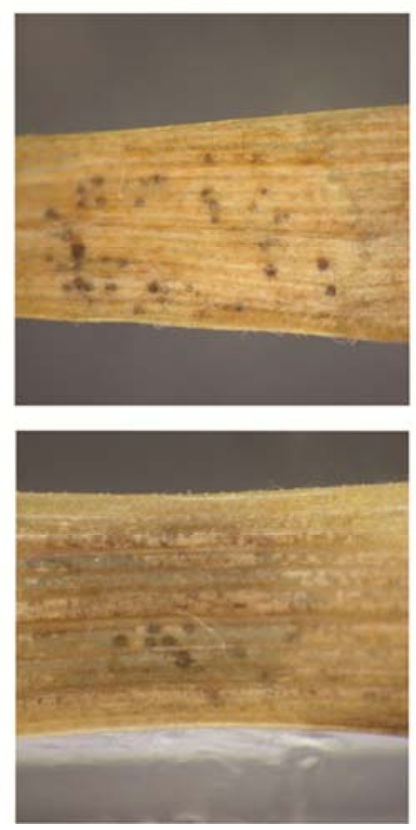

H15
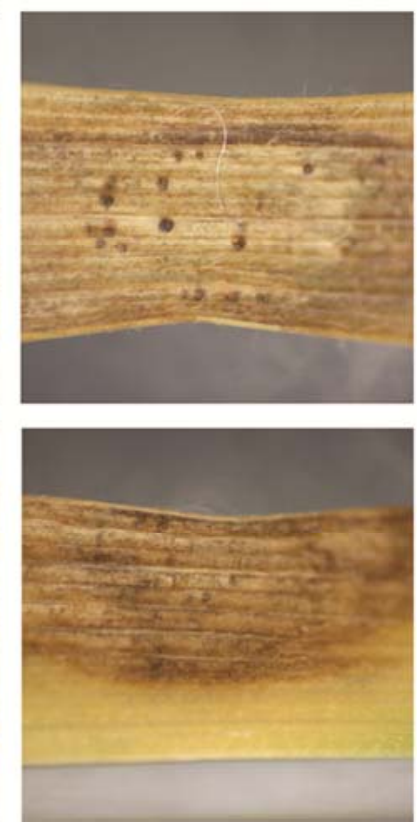

Fig. 3. Reconstituted virulence assay of toxA on Tsn1-positive ('BG261') and Tsn1-negative ('BR34') wheat varieties infiltrated with ToxA H4, H9, and H15 at $200 \mu \mathrm{g} / \mathrm{ml}$. Water was used as a negative infiltration control. No infection and sporulation was observed on wheat leaves mock inoculated with $0.02 \%$ (vol/vol) Tween 20 (data not shown). 
identical (Faris et al. 2010). Therefore, the differences in sensitivity must be due to other factors. These could be different levels of expression of the gene or differential action of modifying genes in the different genetic backgrounds. Gene expression analysis or genetic analysis of a cross between Glenlea and ND2709 could differentiate these and other possibilities.

A major goal in this study was to investigate the functional and evolutionary importance of the excess of $\mathrm{dN} / \mathrm{dS}$ codon changes in the ToxA gene reported earlier in S. nodorum and $P$. tritici-repentis (Stukenbrock and McDonald 2007). High $\mathrm{dN} / \mathrm{dS}$ ratios have been cited as evidence of diversifying selection associated with one-to-one protein-protein effector or receptor interactions leading to a co-evolutionary arms race characterized by alternating qualitative recognition and camouflage (Dodds et al. 2006; Ellis et al. 2008; Ma and Guttman 2008; Meyers et al. 2005). However, all known bread-wheat Tsn1 sources encode identical proteins and we have now shown that all ToxA isoforms cause necrosis in all Tsn1 wheat lines. Furthermore, although ToxA has been reported to interact with ToxABP and plastocyanin, no relevant sequence variation has been observed in these genes (Manning et al. 2007; Tai et al. 2007). If ToxA is undergoing diversifying selection in a manner analogous to that observed in biotrophs, the coevolving partner would have to be an as-yet-unidentified interactor that we predict will also exhibit high $\mathrm{dN} / \mathrm{dS}$ ratios.

Given the lack of diversity in Tsn1, we favor an alternative explanation for the high $\mathrm{dN} / \mathrm{dS}$ ratio, in which ToxA is undergoing selection favoring mutations that increase virulence in regions where Tsn 1 wheat is common. In the presence of Tsn 1 wheat, mutations that increase ToxA virulence would increase in frequency and displace less-virulent alleles if they confer a selective advantage such as the increased sporulation observed for more active ToxA isoforms such as $\mathrm{H} 2$ and $\mathrm{H} 4$. In the case of $P$. tritici-repentis, the time since ToxA was acquired (approximately 70 years ago) (Friesen et al. 2006) may have been insufficient for these mutations to become detectable in the populations we have sampled. Though the ToxA isoform from P. tritici-repentis had the lowest activity, ToxA remains the most significant known determinant of tan spot severity. This indicates that presence of this mild form of the effector was sufficient to imbue $P$. tritici-repentis with the virulence necessary to achieve its current economic importance. The two most potent isoforms, $\mathrm{H} 2$ and $\mathrm{H} 4$, were found only in South Africa, where it is hypothesized that $S$. nodorum acquired the ToxA gene and, thus, where selection has operated for the longest time (Stukenbrock and McDonald 2007). If selection favors more potent isoforms of ToxA, we predict that new, more virulent isoforms will emerge in both pathogens unless and until Tsn1 alleles are eliminated from commonly used wheat cultivars.

\section{MATERIALS AND METHODS}

\section{Plant materials and growth conditions.}

The Brazilian hard red spring wheat breeding line BR34, the North Dakota variety Grandin, and a recombinant inbred line expressing just Tsn1 (BG261) have been described (Friesen et al. 2006). All other wheat cultivars were obtained from G. Grimes, Department of Primary Industries, NSW, Australia. Pots $(10 \mathrm{~cm}$ in diameter) containing perlite (P500) and a grade-2 vermiculite (The Perlite \& Vermiculite Factory, Perth, Australia) were seeded with 20 seeds of each wheat variety and grown at $21^{\circ} \mathrm{C}$ in a 12-h day-and-night cycle in a growth chamber.

\section{Construction of expression cassettes for ToxA isoforms.}

Genomic DNA was purified from isolates of $S$. nodorum as described previously (McDonald et al. 1994). Genomic DNA for $P$. tritici-repentis race 2 (isolate 86-124) was provided by
S. Meinhardt, North Dakota State University, Fargo, ND, U.S.A.

ToxAF and ToxAR primers (Supplementary Table S1) were designed based on the 540-bp partial genomic sequences for SnToxA and PtrToxA. The 5' primer ToxAF included an NdeI site for in-frame cloning, and the $3^{\prime}$ primer ToxAR included an EcoRI site. All sequences amplified contained a predicted intron of $51 \mathrm{bp}$ but did not include the 16-amino-acid signal peptide proposed by Ciuffetti and associates (1997). The template DNA (200 ng) was used in a $50-\mu l$ polymerase chain reaction (PCR) reaction that contained 0.5 units of DyNAzyme EXT DNA polymerase (Finnzymes, Espoo, Finland), $1 \times$ reaction buffer, $1.5 \mathrm{mM}$ final concentration of $\mathrm{MgCl}_{2}$ supplied by the manufacturer, $0.3 \mu \mathrm{M}$ each primer, and $200 \mu \mathrm{M}$ each dNTPs (Promega Corp., Madison, WI, U.S.A.). The PCR conditions consisted of 1 cycle of $94^{\circ} \mathrm{C}$ for 2 min followed by 35 cycles of denaturation for $1 \mathrm{~min}$ at $94^{\circ} \mathrm{C}$, annealing at $1 \mathrm{~min}$ at $65^{\circ} \mathrm{C}$, and extension for $1 \mathrm{~min}$ at $72^{\circ} \mathrm{C}$. The last of the 35 cycles ended with a 5 -min extension at $72^{\circ} \mathrm{C}$. All reactions were carried out in an Eppendorf thermocycler. The desired PCR products $(540 \mathrm{bp})$ were individually ligated into pCR2.1 (Invitrogen, San Diego, CA, U.S.A.). Ligation products were used to transform One-shot TOP10F' chemically competent cells to ampicillin resistance according to the manufacturer's instructions (Invitrogen). Clones (pCRSnToxA-H1gDNA, pCRSnToxA-H3gDNA, pCRSnToxA-H4gDNA, pCRSnToxAH5gDNA, pCRSnToxA-H6gDNA, pCRSnToxA-H7gDNA, pCRSnToxA-H10gDNA, and pCRPtrToxAgDNA) were confirmed by sequencing using an ABI 3730 capillary sequencer (Applied Biosystems, Carlsbad, CA, U.S.A.).

After the initial PCR reactions, double-overlap extension PCR technique (Horton and Pease 1991) was employed to remove the 51-bp intron sequence from the PtrToxA sequence using plasmid DNA as a template with primer combinations ToxAF and PtrTox2AR and PtrTox2AF and ToxAR. Similarly, the 51-bp intron sequence was removed from SnToxA haplotype sequences using different primer combinations. H4, H5, H6, and $\mathrm{H} 2$ sequences for SnToxA were amplified using primer combinations ToxAF and SnToxAH1R and SnToxAH1F and ToxAR. All PCR products were ligated into PCR2.1 (Invitrogen) and clones were sequenced. Appropriate clones were double digested with EcoRI and NdeI and directionally ligated into the pET21c(+) vector (Novagen, Madison, WI, U.S.A.). Ligation products were transformed into E. coli BL21 (DE3) (Novagen).

Plasmid DNAs were used as templates to generate other haplotype sequences (H13, H8, H12, and H9) of SnToxA. Primers ToxAF, SnToxAH8R, SnToxAH8F, and ToxAR (Table 4) were used in double-overlap extension PCR reactions to create sequence $\mathrm{H} 8$ from the PCR template sequence H5 and, similarly, $\mathrm{H} 13$ by altering A to $\mathrm{T}$ at $487 \mathrm{bp}$. The PCR products were ligated into pCR2.1 to create pCRSnToxA-H8 and pCRSnToxA-H13. The final clone, pCRSnToxA-H9, was created with primers ToxAF, SnToxAH9R, SnToxAH9F, and ToxAR (Table 1). Finally, primers ToxAF, SnToxAH12R, SnToxAH12F, and ToxAR (Table 1) were used in PCR reactions designed to change $\mathrm{C}$ to $\mathrm{T}$ at $102 \mathrm{bp}$. The resulting clone, pCRSnTox-H12, was amplified using SnToxA-H6 as the PCR template. Clones were sequenced in both directions to confirm the presence of the desired mutation and the absence of extraneous, nonspecific mutations. Once identified, the correct clones were double digested with EcoRI and NdeI and directionally ligated into the pET $21 \mathrm{c}(+)$ vector (Novagen). All ToxA genes were engineered to produce proteins containing a $6 x \mathrm{His}-$ tag at the $\mathrm{C}$ terminal for the purpose of downstream purification. Ligation products were transformed into E. coli BL21 (DES) (Novagen). 
Protein expression and total cell protein extractions.

ToxA expression was carried out according to the $\mathrm{pET}$ system manual (Novagen) supplied with the BL21(DE3) competent cells, with a final induction of $20 \mathrm{~h}$ at $1 \mathrm{mM}$ (isopropyl-thiogalactopyranoside). After induction, cells were lysed using the Novagen Bugbusters protein extraction reagent. The protein was dissolved from inclusion bodies using $8 \mathrm{M}$ urea as described in the manufacturer's instructions. Briefly, protein purification was performed under denaturing conditions via chromatography using the Novagen Ni-NTA resin kit. The protein was then refolded in buffers with decreasing urea concentrations via dialysis. The final refolding buffer consisted of $250 \mathrm{mM}$ sodium chloride, $100 \mathrm{mM}$ sodium phosphate ( $\mathrm{pH} 8.0), 1 \mathrm{mM}$ EDTA, 1 $\mathrm{mM}$ Tris, $4 \%$ (wt/vol) glycerol and $0.005 \%$ (wt/vol) Tween 20 . Sodium dodecyl sulfate polyacrylamide gel electrophoresis (SDS-PAGE) was performed on a mini-protean III vertical gel apparatus (Bio-Rad Laboratories, Hercules, CA, U.S.A.). Proteins were resolved with a $16 \%$ precast polyacrylamide tricine peptide separation gel (NuSep, Sydney, Australia) using the Tris-tricine buffer system (Schägger and von Jagow 1987). Precision plus and polypeptide SDS-PAGE molecular weight standards (Bio-Rad) were used as protein molecular weight standards. Gels were fixed and visualized via Coomassie G250 colloidal staining (Neuhoff et al. 1988).

\section{Effector bioassay.}

The bioassay was used to characterize the response of varying wheat lines to the different isoforms of ToxA pro-protein obtained from expression of ToxA clones in E. coli. A fully expanded secondary leaf on 2-week-old wheat was infiltrated with ToxA at $25 \mu \mathrm{g} / \mathrm{ml}$. All solutions were applied using a 1-ml syringe with the needle removed. The boundaries of the infiltration site were marked with a nontoxic felt pen prior to the watersoaking disappearing. Plants were kept in growth chambers at $21{ }^{\circ} \mathrm{C}$ with a 12 -h photoperiod. Leaves were evaluated 5 days (unless stated) postinfiltration; a score of 0 indicates no observable symptoms, 1 indicates mild chlorosis, 2 indicates distinct chlorosis, 3 indicates distinct chlorosis with slight necrosis, and 4 indicates full necrosis. All experiments were repeated at least three times, with each experiment giving consistent results.

\section{Reconstituted virulence assay.}

Leaves infiltrated with ToxA H4, H9, and $\mathrm{H} 15$ isoforms at concentrations of 80 to $200 \mu \mathrm{g} / \mathrm{ml}$ were harvested after $24 \mathrm{~h}$ and placed on $1 \%(\mathrm{wt} / \mathrm{vol})$ agar medium containing benzimidazole at $0.75 \mathrm{mg} / \mathrm{liter}$. The infiltration zones were inoculated with 5,000 spores in $0.02 \%$ (vol/vol) Tween 20 of $S$. nodorum toxA just below the point of infiltration to avoid any damaged area as a result of the syringe. The assay was left to develop for 5 days in a $25^{\circ} \mathrm{C}$ growth room with a 12-h photoperiod. The number of pycnidia on the infected leaves at each stage of development was counted under a dissecting microscope (Supplementary Fig. S4) (Halama et al. 1992; Solomon et al. 2006b). The composite virulence score for each treatment was calculated using the formula virulence score $=[9 \times($ stage 5$)]+[3 \times$ (stage 3 and 4$)]+[1 \times($ stage 1 and 2$)]$.

\section{CD spectroscopy.}

ToxA isoforms were diluted to $0.1 \mathrm{mg} / \mathrm{ml}$ in $10 \mathrm{mM}$ potassium phosphate, $\mathrm{pH} 7.0$ and subjected to CD spectroscopy. Each spectrum was measured using a Jasco J810 spectropolarimeter (Jasco Inc., Easton, MD, U.S.A.), using a 0.1-cmpath-length quartz cuvette at $25^{\circ} \mathrm{C}$ with a bandwidth of $1 \mathrm{~nm}$ and spectral range of 190 to $250 \mathrm{~nm}$. Five spectra were accumulated for each sample and three samples of each ToxA isoforms were analyzed. Buffer background was subtracted from each spectrum prior to further analysis.
Secondary structure estimations were conducted using the Dichroweb interface (Whitmore and Wallace 2008). Each spectra was analyzed using CONTIN-LL (van Stokkum et al. 1990) and reference data set 4 . Secondary structure means and standard deviations were calculated from analysis of the three spectra obtained for each ToxA isoforms. Secondary structure content from the X-ray crystal structure of PtrToxA (PDB code 1ZLD) (Sarma et al. 2005) was assigned with STRIDE (Frishman and Argos 1995).

\section{Statistical analysis.}

All statistical analyses were performed with the JMP 7 software (SAS Institute, Cary, NC, U.S.A.). Analysis of variance employing the Tukey-Kramer test was used for all statistical analyses.

\section{ACKNOWLEDGMENTS}

This work was supported by grants from the Australian Grains Research and Development Corporation. ToxA sequences were obtained in the Genetic Diversity Center at ETH Zurich.

\section{LITERATURE CITED}

Abeysekara, N. S., Friesen, T. L., Keller, B., and Faris, J. D. 2009. Identification and characterization of a novel host-toxin interaction in the wheat-Stagonospora nodorum pathosystem. Theor. Appl. Genet. 1-10.

Abramovitch, R. B., Anderson, J. C., and Martin, G. B. 2006. Bacterial elicitation and evasion of plant innate immunity. Nat. Rev. Mol. Cell Biol. 7:601-611.

Aguilar, V., Stamp, P., Winzeler, M., Winzeler, H., Schachermayr, G. Keller, B., Zanetti, S., and Messmer, M. M. 2005. Inheritance of field resistance to Stagonospora nodorum leaf and glume blotch and correlations with other morphological traits in hexaploid wheat (Triticum aestivum L.). Theor. Appl. Genet. 111:325-336.

Allen, R. L., Bittner-Eddy, P. D., Grenville-Briggs, L. J., Meitz, J. C., Rehmany, A. P., Rose, L. E., and Beynon, J. L. 2004. Host-parasite coevolutionary conflict between Arabidopsis and downy mildew. Science 306:1957-1960

Chisholm, S. T., Coaker, G., Day, B., and Staskawicz, B. J. 2006. Hostmicrobe interactions: Shaping the evolution of the plant immune response. Cell 124:803-814.

Ciuffetti, L. M., Tuori, R. P., and Gaventa, J. M. 1997. A single gene encodes a selective toxin causal to the development of tan spot of wheat. Plant Cell 9:135-144.

De Wit, P. J., Mehrabi, R., Van den Burg, H. A., and Stergiopoulos, I. 2009. Fungal effector proteins: Past, present and future. Mol. Plant Pathol. 10:735-747.

Dodds, P. N., Lawrence, G. J., Catanzariti, A. M., The, T., Wang, C. I., Ayliffe, M. A., Kobe, B., and Ellis, J. G. 2006. Direct protein interaction underlies gene-for-gene specificity and coevolution of the flax resistance genes and flax rust avirulence genes. Proc. Natl. Acad. Sci. U.S.A. 103:8888-8893.

Dodds, P. N., Rafiqi, M., Gan, P. H., Hardham, A. R., Jones, D. A., and Ellis, J. G. 2009. Effectors of biotrophic fungi and oomycetes: Pathogenicity factors and triggers of host resistance. New Phytol. 183:9931000 .

Ellis, J., Catanzariti, A. M., and Dodds, P. 2006. The problem of how fungal and oomycete avirulence proteins enter plant cells. Trends Plant Sci. 11:61-63.

Ellis, J. G., Dodds, P. N., and Lawrence, G. J. 2008. Flax rust resistance gene specificity is based on direct resistance-avirulence protein interactions. Annu. Rev. Phytopathol. 45:289-306.

Faris, J. D., and Friesen, T. L. 2009. Reevaluation of a tetraploid wheat population indicates that the Tsnl-ToxA interaction is the only factor governing Stagonospora nodorum blotch susceptibility. Phytopathology 99:906-912.

Faris, J. D., Anderson, J. A., Francl, L. J., and Jordahl, J. G. 1996. Chromosomal location of a gene conditioning insensitivity in wheat to a necrosis-inducing culture filtrate from Pyrenophora tritici-repentis. Phytopathology 86:459-463.

Faris, J. D., Zhang, Z., Lu, H., Lu, S., Reddy, L., Cloutier, S., Fellers, J. P., Meinhardt, S. W., Rasmussen, J. B., Xu, S. S., Oliver, R. P., Simons, K. J., and Friesen, T. L. 2010. A unique wheat disease resistance-like gene governs effector-triggered susceptibility to necrotrophic pathogens. Proc. Natl. Acad. Sci. U.S.A. 107:13544-13549. 
Friesen, T. L., Rasmussen, J. B., Kwon, C. Y., Ali, S., Francl, L. J., and Meinhardt, S. W. 2002. Reaction of Ptr ToxA-insensitive wheat mutants to Pyrenophora tritici-repentis race 1. Phytopathology 92:38-42.

Friesen, T. L., Ali, S., Kianian, S., Francl, L. J., and Rasmussen, J. B. 2003. Role of host sensitivity to Ptr ToxA in development of tan spot of wheat. Phytopathology 93:397-401.

Friesen, T. L., Stukenbrock, E. H., Liu, Z., Meinhardt, S., Ling, H., Faris, J. D., Rasmussen, J. B., Solomon, P. S., McDonald, B. A., and Oliver, R. P. 2006. Emergence of a new disease as a result of interspecific virulence gene transfer. Nat. Genet. 38:953-956.

Friesen, T. L., Meinhardt, S. W., and Faris, J. D. 2007. The Stagonospora nodorum-wheat pathosystem involves multiple proteinaceous hostselective toxins and corresponding host sensitivity genes that interact in an inverse gene-for-gene manner. Plant J. 51:681-692.

Friesen, T. L., Faris, J. D., Solomon, P. S., and Oliver, R. P. 2008. Hostspecific toxins: Effectors of necrotrophic pathogenicity. Cell. Microbiol. 10:1421-1428.

Friesen, T. L., Chu, C. G., Liu, Z. H., Xu, S. S., Halley, S., and Faris, J. D. 2009. Host-selective toxins produced by Stagonospora nodorum confer disease susceptibility in adult wheat plants under field conditions. Theor. Appl. Genet. 118:1489-1497.

Frishman, D., and Argos, P. 1995. Knowledge-based protein secondary structure assignment. Proteins 23:566-579.

Halama, P., Parguey-Leduc, A., and Lacoste, L. 1992. Les organes de reproduction de l'ascomycète Phaeosphaeria (Leptosphaeria) nodorum, et de la forme asexuée Septoria nodorum. Can. J. Bot. 70:1401-1408.

Horton, R. M., and Pease, L. R. 1991. Recombination and mutagenesis of DNA sequences using PCR. Pages 217-247 in: Directed Mutagenesis: A Practical Approach. M. J. McPherson, ed. Oxford University Press, Oxford.

Jones, J. D. G., and Dangl, J. L. 2006. The plant immune system. Nature 444:323-329.

Lamari, L., Gilbert, J., and Tekauz, A. 1998. Race differentiation in Pyrenophora tritici-repentis and survey of physiologic variation in western Canada. Can. J. Plant Pathol. 20:396-400.

Leach, J., Tegtmeier, K., Daly, J., and Yoder, O. 1982. Dominance at the Tox1 locus controlling T-toxin production by Cochliobolus heterostrophus. Physiol. Plant Pathol. 21:327-333.

Lewis, D. H. 1973. Concepts in fungal nutrition and the origin of biotrophy. Biol. Rev. 48:261-278.

Liu, Z., Friesen, T. L., Ling, H., Meinhardt, S. W., Oliver, R. P., Rasmussen, J. B., and Faris, J. D. 2006. The Tsn1-ToxA interaction in the wheatStagonospora nodorum pathosystem parallels that of the wheat-tan spot system. Genome 49:1265-1273.

Liu, Z., Faris, J. D., Oliver, R. P., Tan, K.-C., Solomon, P. S., McDonald, M. C., McDonald, B. A., Nunez, A., Lu, S., Rasmussen, J. B., Friesen, T. L.2009. SnTox3 acts in effector triggered susceptibility to induce disease on wheat carrying the Snn3 gene. PLoS Pathog. 5. Published online.

Liu, Z. H., Faris, J. D., Meinhardt, S. W., Ali, S., Rasmussen, J. B., and Friesen, T. L. 2004a. Genetic and physical mapping of a gene conditioning sensitivity in wheat to a partially purified host-selective toxin produced by Stagonospora nodorum. Phytopathology 94:1056-1060.

Liu, Z. H., Friesen, T. L., Rasmussen, J. B., Ali, S., Meinhardt, S. W., and Faris, J. D. 2004b. Quantitative trait loci analysis and mapping of seedling resistance to Stagonospora nodorum leaf blotch in wheat. Phytopathology 94:1061-1067.

Lorang, J. M., Sweat, T. A., and Wolpert, T. J. 2007. Plant disease susceptibility conferred by a "resistance" gene. Proc. Natl. Acad. Sci. U.S.A. 104:14861-14866.

Ma, W., and Guttman, D. S. 2008. Evolution of prokaryotic and eukaryotic virulence effectors. Curr. Opin. Plant Biol. 11:412-419.

Manning, V. A., and Ciuffetti, L. M. 2005. Localization of Ptr ToxA produced by Pyrenophora tritici-repentis reveals protein import into wheat mesophyll cells. Plant Cell 17:3203-3212.

Manning, V. A., Andrie, R. M., Trippe, A. F., and Ciuffetti, L. M. 2004. Ptr ToxA requires multiple motifs for complete activity. Mol. Plant-Microbe Interact. 17:491-501.

Manning, V. A., Hardison, L. K., and Ciuffetti, L. M. 2007. Ptr ToxA interacts with a chloroplast-localized protein. Mol. Plant-Microbe Interact. 20:168-177.

McDonald, B. A., Miles, J., Nelson, L. R., and Pettway, R. E. 1994. Genetic variability in nuclear DNA in field populations of Stagonospora nodorum. Phytopathology 84:250-255.

Meinhardt, S. W., Cheng, W., Kwon, C. Y., Donohue, C. M., and Rasmussen, J. B. 2002. Role of the arginyl-glycyl-aspartic motif in the action of Ptr ToxA produced by Pyrenophora tritici-repentis. Plant Physiol. 130:1545-1551.

Meyers, B. C., Kaushik, S., and Nandety, R. S. 2005. Evolving disease resistance genes. Curr. Opin. Plant Biol. 8:129-134.

Nagy, E. D., and Bennetzen, J. L. 2008. Pathogen corruption and sitedirected recombination at a plant disease resistance gene cluster. Genome Res. 18:1918-1923.

Neuhoff, V., Arold, N., Taube, D., and Ehrhardt, W. 1988. Improved staining of proteins in polyacrylamide gels including isoelectric focusing gels with clear background at nanogram sensitivity using Coomassie Brilliant Blue G-250 and R-250. Electrophoresis 9:255-262.

Oliver, R. P. 2008. Plant breeding for disease resistance in the age of effectors. Phytoparasitica 37:1-5.

Oliver, R. P., and Ipcho, S. V. S. 2004. Arabidopsis pathology breathes new life into the necrotrophs-vs.-biotrophs classification of fungal pathogens. Mol. Plant Pathol. 5:347-352.

Oliver, R. P., and Solomon, P. S. 2010. New developments in pathogenicity and virulence of necrotrophs. Curr. Opin. Plant Biol. 13:415-419.

Oliver, R. P., Lord, M., Rybak, K., Faris, J. D., and Solomon, P. S. 2008. Emergence of tan spot disease caused by toxigenic Pyrenophora triticirepentis in Australia is not associated with increased deployment of toxin-sensitive cultivars. Phytopathology 98:488-491.

Oliver, R. P., Rybak, K., Solomon, P. S., and Ferguson-Hunt, M. 2009. Prevalence of ToxA-sensitive alleles of the wheat gene Tsn1 in Australian and Chinese wheat cultivars. Crop Pasture Sci. 60:348-352.

Parlange, F., Daverdin, G., Fudal, I., Kuhn, M. L., Balesdent, M. H., Blaise, F., Grezes-Besset, B., and Rouxel, T. 2009. Leptosphaeria maculans avirulence gene AvrLm4-7 confers a dual recognition specificity by the Rlm4 and Rlm7 resistance genes of oilseed rape, and circumvents Rlm4-mediated recognition through a single amino acid change. Mol. Microbiol. 71:851-863.

Poland, J. A., Balint-Kurti, P. J., Wisser, R. J., Pratt, R. C., and Nelson, R. J. 2009. Shades of gray: The world of quantitative disease resistance. Trends Plant Sci. 14:21-29.

Rasmussen, J. B., Kwon, C. Y., and Meinhardt, S. W. 2004. Requirement of host signaling mechanisms for the action of Ptr ToxA in wheat. Eur. J. Plant Pathol. 110:333-335.

Sarma, G. N., Manning, V. A., Ciuffetti, L. M., and Karplus, P. A. 2005. Structure of Ptr ToxA: An RGD-containing host-selective toxin from Pyrenophora tritici-repentis. Plant Cell 17:3190-3202.

Schägger, H., and von Jagow, G. 1987. Tricine-sodium dodecyl sulfatepolyacrylamide gel electrophoresis for the separation of proteins in the range from 1 to $100 \mathrm{kDa}$. Anal. Biochem. 166:368-379.

Solomon, P. S., Lowe, R. G. T., Tan, K. C., Waters, O. D. C., and Oliver, R. P. 2006a. Stagonospora nodorum: Cause of Stagonospora nodorum blotch of wheat. Mol. Plant Pathol. 7:147-156.

Solomon, P. S., Wilson, T. J. G., Rybak, K., Parker, K., Lowe, R. G. T., and Oliver, R. P. 2006b. Structural characterisation of the interaction between Triticum aestivum and the dothideomycete pathogen Stagonospora nodorum. Eur. J. Plant Pathol. 114:275-282.

Stergiopoulos, I., De Kock, M. J. D., Lindhout, P., and De Wit, P. J. G. M. 2007. Allelic variation in the effector genes of the tomato pathogen Cladosporium fulvum reveals different modes of adaptive evolution. Mol. Plant-Microbe Interact. 20:1271-1283.

Stukenbrock, E. H., and McDonald, B. A. 2007. Geographical variation and positive diversifying selection in the host-specific toxin SnToxA Mol. Plant Pathol. 8:321-332.

Sweat, T. A., Lorang, J. M., Bakker, E. G., and Wolpert, T. J. 2008. Characterization of natural and induced variation in the LOV1 gene, a CCNB-LRR gene conferring victorin sensitivity and disease susceptibility in Arabidopsis. Mol. Plant-Microbe Interact. 21:7-19.

Tai, Y. S., Bragg, J., and Meinhardt, S. W. 2007. Functional characterization of ToxA and molecular identification of its intracellular targeting protein in wheat. Am. J. Plant Physiol. 2:76-89.

Tuori, R. P., Wolpert, T. J., and Ciuffetti, L. M. 2000. Heterologous expression of functional Ptr ToxA. Mol. Plant-Microbe Interact. 13:456-464.

van Stokkum, I. H., Spoelder, H. J., Bloemendal, M., van Grondelle, R., and Groen, F. C. 1990. Estimation of protein secondary structure and erro analysis from circular dichroism spectra. Anal. Biochem. 191:110-118.

Whitmore, L., and Wallace, B. A. 2008. Protein secondary structure analyses from circular dichrosim spectroscopy; methods and reference databases. Biopolymers 89:392-400.

Wolpert, T. J., Dunkle, L. D., and Ciuffetti, L. M. 2002. Host-selective toxins and avirulence determinants: What's in a name? Annu. Rev. Phytopathol. 40:251-285.

Yoder, O. 1980. Toxins in plant pathogenesis. Annu. Rev. Phytopathol. 18:103-129.

Zhang, Z., Friesen, T. L., Xu, S. S., Shi, G., Liu, Z., Rasmussen, J. B., and Faris, J. D. 2011. Two putatively homoeologous wheat genes mediate recognition of SnTox3 to confer effector-triggered susceptibility to Stagonospora nodorum. Plant J. 65:27-38. 